\title{
Shape Extraction Via Heat Flow Analogy
}

\author{
Cem Direkoğlu and Mark S. Nixon \\ Department of Electronics and Computer Science \\ University of Southampton \\ Southampton SO17 1BJ, UK \\ \{cd05r, msn $\}$ decs. soton.ac.uk
}

\begin{abstract}
In this paper, we introduce a novel evolution-based segmentation algorithm by using the heat flow analogy, to gain practical advantage. The proposed algorithm consists of two parts. In the first part, we represent a particular heat conduction problem in the image domain to roughly segment the region of interest. Then we use geometric heat flow to complete the segmentation, by smoothing extracted boundaries and removing possible noise inside the prior segmented region. The proposed algorithm is compared with active contour models and is tested on synthetic and medical images. Experimental results indicate that our approach works well in noisy conditions without pre-processing. It can detect multiple objects simultaneously. It is also computationally more efficient and easier to control and implement in comparison to active contour models.
\end{abstract}

\section{Introduction}

There are two main types of image segmentation methods that evolve to the target solution: active contours and region growing techniques. We first review these techniques with special consideration of their advantages and practical limitations. We then describe techniques which are based on the use of the heat flow analogy, including the proposed model and its advantages as a segmentation technique.

\subsection{Related Works}

Active contours (snakes) are curves that evolve to recover object shapes. Active contours can be classified as Parametric Active contours (PAC) and Geometric Active contours (GAC). The first PAC model was introduced by Kass et al. [1]. In this, segmentation is achieved by using gradient vectors of an edge map. Problems associated with this model are initialization and poor convergence to concave regions. These problems were largely solved with the development of new external force model, which is called the Gradient Vector Flow (GVF) [2]. GVF is computed as a diffusion of the gradient vectors of an edge map. However, PAC models can have difficulty with simultaneous detection of multiple objects, because of the explicit representation of curve. To solve this problem, GAC models have been introduced, where the curve is represented implicitly in a level set function. Caselles et al. [3] and Malladi et al. [4] proposed the first GAC model, which uses gradient based information for 
segmentation. The gradient based GAC can detect multiple objects simultaneously but it has other important problems: boundary leakage, noise sensitivity, computational inefficiency and complexity of implementation. In [5], gradient based information has been improved to solve boundary leakage and noise sensitivity problems. However, it can just increase the tolerance, since gradient based information is always limited by noise. Several numerical schemes have also been proposed to improve the computational efficiency of the level set method, including narrow band [6], fast marching [7] and additive operator splitting [8]. Despite substantial improvements in efficiency, they are still not effective enough and can be difficult to implement. Chen and Vese [9] introduced a new GAC model based on the Mumford-Shah functional [10]. Their model uses regional statistics for segmentation. This model is good at initialization, handling noise and boundary leaking but still suffers from computational complexity and from difficulty of implementation, because of the level set method.

Region growing is a procedure that groups pixels or sub-regions into larger regions based on predefined similarity criteria for region growth. The basic approach starts with a seed point and merges neighboring pixels that have pre-defined properties similar to the seed, such as intensity [11] or texture [12]. Although, region growing techniques can detect multiple objects simultaneously and can be more efficient than active contour models, the main problem is selection of the similarity criteria. They also have to use connectivity information to define the neighboring pixels in each step of growth. In addition, they can achieve region segmentation with irregular boundaries and holes in the presence of high noise, since they omit smoothing.

\subsection{Heat Flow in Image Processing and Computer Vision}

The heat flow analogy has been used for image smoothing and enhancement [13] [14]. Anisotropic diffusion, which was introduced to vision by Perona and Malik [13], is the state-of-art image enhancement technique. In motion analysis, we can also see a significant application of heat flow by [15]. The algorithm combines anisotropic and isotropic heat flow to obtain moving edges. In [16], an anisotropic diffusion pyramid was introduced for region based segmentation. The pyramid is constructed using the scale space representation of the anisotropic diffusion. In [17], the anti-geometric heat flow model was introduced for the segmentation of regions. Here, anti-geometric heat flow is represented as diffusion through the normal direction of edges.

In this paper, we introduce a novel segmentation algorithm based on the heat flow analogy. The proposed algorithm consists of two parts. In the first part, we represent a particular heat conduction problem in the image domain to roughly segment objects of interest. In this problem, we consider a conductive solid body with initial and boundary conditions respectively given by $T(\mathbf{x}, t=0)=0$ and $T(\mathbf{x}, t)=0$, where $T$ represents the temperature at position $\mathbf{x}=(x, y)$ and time $t$. The given conditions mean that the temperature is initially zero inside the body and the boundary condition is "Dirichlet" that has specified temperature, zero, at the boundary layer all the time. If we initialize a continuous heat source, which is a positive constant, at any point inside the body, there will be heat diffusion to the other points from the source position as time passes and this will cause temperature increase in the body except at the boundary layer. This concept is represented in the image domain by using a control function 
in the heat conduction equation. The control function is obtained from the source located region statistics, since we propose to segment the source located region. However, in noisy conditions, we can observe irregular boundaries and holes inside the segmented region. These problems are solved in the second part of the algorithm, which is geometric heat flow. In this part, the segmented image is firstly converted to binary form and then geometric heat flow is applied to reduce curvature in the boundary, as well as to remove holes inside the segmented region. After a specified number of iterations, the resultant image is thresholded and the final segmentation is obtained. Experimental results indicate that the proposed algorithm works well in noisy conditions without pre-processing. It can detect multiple objects simultaneously. It is also computationally more efficient and easier to control and implement in comparison to active contour models. As such, by using physics based analogies, we can control the segmentation process so as to achieve a result which offers improved segmentation, by a better fit to the image data.

The rest of the paper is organized as follows: Section 2 explains the basic concepts of heat flow. Section 3 represents the proposed heat conduction problem in the image domain. Section 4 discusses the geometric heat flow. Section 5 concerns evaluation and experimental results and finally Section 6 is conclusions. A List of acronyms is also given below in Table 1 .

Table 1. List of Acronyms

\begin{tabular}{ll}
\hline ACWE & Active Contours Without Edges \\
CF & Control Function \\
GAC & Geometric Active Contours \\
GHF & Geometric Heat Flow \\
GVF & Gradient Vector Flow \\
GVFS & Gradient Vector Flow Snake \\
PAC & Parametric Active Contours \\
TF & Temperature Front \\
\hline
\end{tabular}

\section{Basic Concepts of Heat Flow}

Conduction, convection and radiation are three different modes of heat flow. Here, we chose to investigate use of a conduction model, which we found to operate well. Conduction is the flow of heat energy from high- to low-temperature regions due to the presence of a thermal gradient in a body [18]. The change of temperature over time at each point of material is described using the general heat conduction or diffusion equation,

$$
d T / d t=\alpha\left(d^{2} T / d x^{2}+d^{2} T / d y^{2}\right)+Q=\alpha \nabla^{2} T+Q
$$

Where, $\nabla$ represents gradient, $\alpha$ is called thermal diffusivity of the material and a larger values of $\alpha$ indicate faster heat diffusion through the material. $Q$ is the source term that applies internal heating. It can be uniformly or non-uniformly distributed over material body. The solution of this equation provides the temperature distribution 
over the material body and it depends on time, distance, heat source, properties of material, as well as specified initial and boundary conditions.

Initial conditions specify the temperature distribution in a body, as a function of space coordinates, at the origin of the time coordinate $(t=0)$. Initial conditions are represented as follows,

$$
T(\mathbf{x}, t=0)=\Phi(\mathbf{x})
$$

where, $\mathbf{x}=(x, y)$ is the space vector for the two-dimensional case and $\Phi(\mathbf{x})$ is the function that specifies the initial temperature inside the body.

Boundary conditions specify the temperature or the heat flow at the boundaries of the body. There are three general types of boundary conditions: Dirichlet, Neuman and Robin. Here, we explain the Dirichlet condition, which is used in our algorithm. In the Dirichlet condition, temperature is specified along the boundary layer. It can be a function of space and time, or constant. The Dirichlet condition is represented as follows,

$$
T(\mathbf{x}, t)=f(\mathbf{x})
$$

where $f(\mathbf{x})$ is the function that specifies the temperature at the boundary layer. Many heat conduction problems do not have analytical solutions. These problems usually involve geometrical shapes that are mathematically unsuited to representing initial and boundary conditions. However, numerical techniques exist, such as finite differences and finite elements, which are able to handle almost all problems with any complex shapes. The numerical methods yield numerical values for temperatures at selected discrete points within the body and only at discrete time intervals.

The numerical heat conduction problem can be investigated in the image domain, since the image is formed by a set of points, as well as since the image is convenient for the finite difference technique. Each object in image can represent bodies and each pixel in object can represent points within the body.

\section{Proposed Heat Conduction Problem and Representation in Image Domain}

Consider a two-dimensional conductive solid body with initial and boundary conditions respectively given by $T(\mathbf{x}, t=0)=0$ and $T(\mathbf{x}, t)=0$, which mean the temperature is initially zero inside the body and the boundary condition is Dirichlet that has specified temperature (zero) at the boundaries. If we initialize a continuous heat source, which is a positive constant, at a point inside the body, there will be heat diffusion to the other points from the source position. As a result of this, all the points inside the body will have temperature values exceeding zero, except the boundary points. This is then an ideal approach for object segmentation in computer images. Let us investigate the proposed problem on a square object that is inside the grey-level image $(G)$, as shown in Fig. 1(a). Assume that all the temperature values of the objects and the background are kept in another image, which is represented by $I$, and the initial condition of whole image is zero, $I(\mathbf{x}, t=0)=0$. This assumption means that all objects have temperature initially zero inside, as well as at the boundaries. When we initialize 
a heat source at any pixel inside the square object, as shown in Fig. 1(a), there will be heat diffusion to the other pixels from the source position, which will cause temperature to increase. However the temperature at the boundary layer must be kept at zero all the time to obtain the Dirichlet condition, where the boundary layer is defined at the external side of an object as shown in Fig. 1(b). To achieve this, we use a control function in the heat conduction equation as given below,

$$
d I(\mathbf{x}, t) / d t=C F(\mathbf{x}, t)\left(\alpha \nabla^{2} I(\mathbf{x}, t)\right)+Q(\mathbf{x})
$$

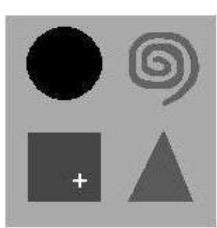

(a)

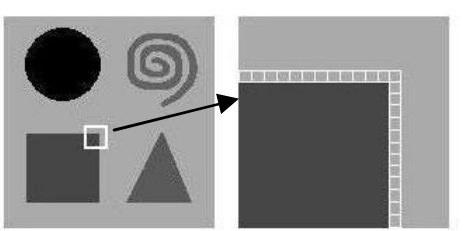

(b)

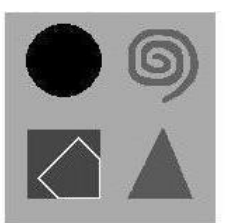

(c)

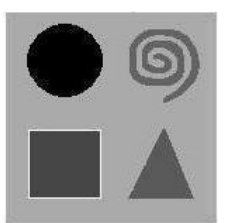

(d)

Fig. 1. Heat conduction modeling in image domain of size $150 \times 150$. (a) Source position at $t=0$. (b) Boundary layer illustration. (c) TF at $t=30$ (iterations). (d) Final TF at $t=72$.

where $I(\mathbf{x}, t)$ represents an image pixel value in terms of temperature at each point and time, $\alpha$ is the thermal diffusivity and $0 \leq \alpha \leq 0.25$ for the numerical scheme to be stable in two-dimensional system [18], $Q(\mathbf{x})$ is the source term and $C F(\mathbf{x}, t)$ is the control function. The control function is obtained from the region statistics of source location on a given grey-level image. The proposed region statistics model is similar to the one used in [9]. In this model, the image is divided into two regions, interior and exterior, separated by contour and the model minimizes the variance inside and outside of the surface of desired object. In our model, the contour is represented by a Temperature Front (TF), where the TF is the boundary of the region that has temperature values exceeding zero. The control function, $C F(\mathbf{x}, t)$, is formulated as follows,

$$
\begin{gathered}
\sigma_{1}(\mathbf{x}, t)=\lambda_{1}\left|G(\mathbf{x})-\mu_{\text {in }}\right|^{2} \\
\sigma_{2}(\mathbf{x}, t)=\lambda_{2}\left|G(\mathbf{x})-\mu_{\text {out }}\right|^{2}
\end{gathered}
$$

where, $\lambda_{1}>0$ and $\lambda_{2}>0$ are fixed parameters for regional statistics, $G(\mathbf{x})$ is the given grey-level image, $\sigma_{1}(\mathbf{x}, t)$ is variance, at each point and time, with respect to the mean, $\mu_{i n}$, inside of the TF and $\sigma_{2}(\mathbf{x}, t)$ is variance, at each point and time, with respect to the mean, $\mu_{\text {out }}$, outside of the TF. Then, the following logical decision is applied in each position and time increment.

$$
C F(\mathbf{x}, t)=\left\{\begin{array}{cc}
1, & \sigma_{1}(\mathbf{x}, t) \leq \sigma_{2}(\mathbf{x}, t) \\
0, & \text { otherwise }
\end{array}\right.
$$

Therefore, the control function allows heat diffusion inside the object of interest and achieves the proposed Dirichlet condition on the boundary layer by keeping the temperature value at zero. However, it is better to start this process after a short diffusion time by assuming $C F(\mathbf{x}, t)=1$ at all points. Because, it will increase the number 
of samples inside of the TF, which means better decision at the first step especially for noisy cases. In addition, the heat source must be initialized onto a smooth surface of the object, since the source localization to the edge pixel will give the wrong region statistic for our purpose. Fig. 1(c) and (d) respectively show the evolution and the final position of the TF. However, there is no need to continue diffusion, after the TF reaches its final position. For this reason, the position of the TF is controlled in each specified time interval and when there is no movement, diffusion is terminated automatically. The main difference between [9] and our model, in using region statistics, we attempt to segment region of source location instead of whole image.

One difficulty arises when the source located region intersect with the image boundary. This problem can be solved by assuming that image is surrounded by a boundary layer, at the external side, which has temperature value zero all the time (Dirichlet). Fig. 2 shows the evolution and the final position of the TF, which has source location at the background. The result, in Fig. 2, also shows that multiple object detection can be achieved and the heat can diffuse through the narrow regions within the spiral object.

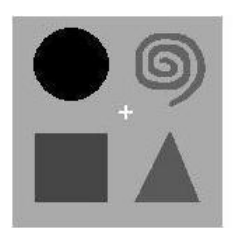

(a) Source position

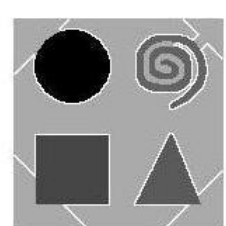

(b) $t=110$

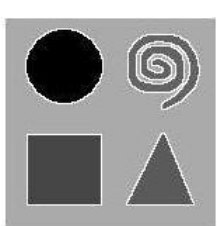

(c) $t=221$ (final)

Fig. 2. TF is moving on background in the image of size $150 \times 150$

It is also required to consider the control function when the given image is bimodal. In this case, the control function attempts to segment the whole image while the TF segments the source located region. The reason for this is that, the control function assigns unity to the pixels that are similar to the inside of the TF, and assigns zero to the pixels that are not similar.

All the results so far have been on synthetic images without added noise. If we simulate this algorithm on noisy medical images, such as human heart image shown in Fig. 3(a) with the heat source location, we observe some drawbacks in segmentation. The drawbacks are irregular boundaries and holes inside the segmented region, as shown in Fig. 3(b). These problems are solved by using the heat flow analogy again as described in the next section.

\section{Geometric Heat Flow}

Geometric Heat Flow (GHF) is a kind of anisotropic diffusion and is widely used for image denoising and enhancement [14]. It diffuses along the boundaries of image features, but not across them. It derives its name from the fact that, under this flow, the feature boundaries of the image evolve in the normal direction in proportion to 
their curvature. Thus, GHF decreases the curvature of shapes while removing noise, in the images. GHF equation is obtained with the following consideration.

Edge directions are related to the tangents of the feature boundaries of an image $B$. Let $\eta$ denote the direction normal to the feature boundary through a given point (the gradient direction), and let $\tau$ denote the tangent direction. Since $\eta$ and $\tau$ constitute orthogonal directions, the rotationally invariant Laplacian operator can be expressed as the sum of the second order spatial derivatives, $B_{\eta \eta}$ and $B_{\tau \tau}$, in these directions and the heat conduction equation can be written without using the source term,

$$
d B / d t=\alpha \nabla^{2} B=\alpha\left(B_{\eta \eta}+B_{\tau \tau}\right)
$$

Omitting the normal diffusion, while keeping the tangential diffusion yields the GHF equation as

$$
\frac{d B}{d t}=\alpha B_{\tau \tau}=\alpha \frac{\left(B_{x x} B_{y}^{2}-2 B_{x y} B_{x} B_{y}+B_{y y} B_{x}^{2}\right)}{\left(B_{x}^{2}+B_{y}^{2}\right)}
$$

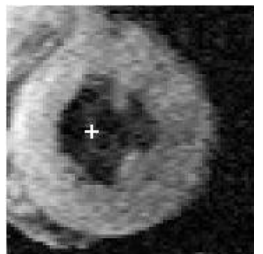

(a) Source position

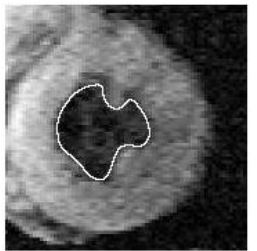

(e) Final shape

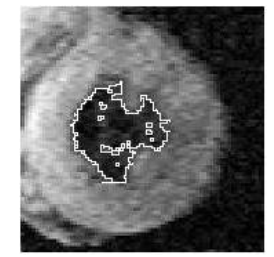

(b) Final TF at $t=59$

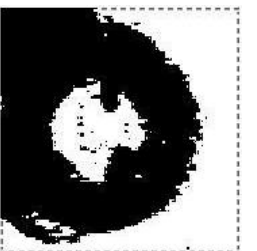

(f) $C F(\mathbf{x})$

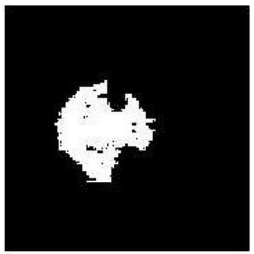

(c) $B(\mathbf{x})$

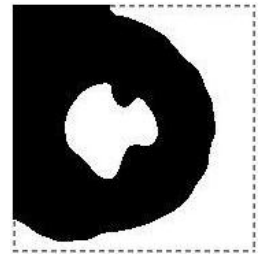

(g) $C F(\mathbf{x})$ after GHF

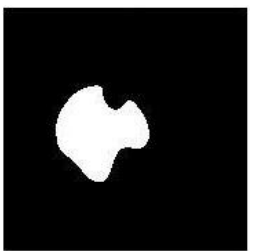

(d) $S(\mathbf{x})$

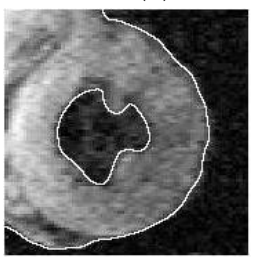

(h) Final shape

Fig. 3. Illustration of GHF for the purpose of obtaining smooth boundaries and removing holes inside the prior segmented regions. GHF is applied both to the binary form of the TF segmentation, $B(\mathbf{x})$, and to the control function $C F(\mathbf{x})$. The size of the human heart image is $177 \times 178$.

In our model, GHF is used to decrease curvature for the purpose of obtaining smooth boundaries and removing holes that appear because of noise. This is achieved as follows. Firstly, a segmented region is converted to a binary form as given below and also shown in Fig. 3(c),

$$
B(\mathbf{x})= \begin{cases}1, & I(\mathbf{x})>0 \\ 0, & I(\mathbf{x})=0\end{cases}
$$

where $I(\mathbf{x})$ is the temperature distribution after terminating diffusion and $B(\mathbf{x})$ is the binary form of the segmented image that assigns unity to the region of interest. Then, 
GHF is applied to the $B(\mathbf{x})$ until the specified time (number of iterations) and finally the resulting image is thresholded to obtain the final segmentation. The process is formulated below,

$$
S(\mathbf{x})= \begin{cases}1, & G H F\left(B(\mathbf{x}), t_{s}\right) \geq 0.5 \\ 0, & G H F\left(B(\mathbf{x}), t_{s}\right)<0.5\end{cases}
$$

where, $t_{s}$ is the number of iterations and $S(\mathbf{x})$ is the binary form of the final segmentation, which assigns unity to the region of interest. The final segmentation is shown in Fig. 3(d) and (e), where $t_{s}=50$ for this illustration. The selection of $t_{s}$ depends on the user and it is determined due to the noise condition of the image. However, as $t_{s}$ increases, the extracted shape evolves to a circle, then to a point and then it is lost. For this reason, we should avoid to use large values for $t_{s}$.

Since the illustrated human heart image seems bimodal, we can also consider the final form of the control function as shown in Fig. 3(f). To smooth boundaries and remove holes, we simply continue with Eq. (11) and observe the result in Fig. 3(g) and (h).

\section{Evaluation and Experimental Results}

In this section, we first present the evaluation of segmentation by $\mathrm{TF}$ and then there are some illustrative examples on medical images.

Segmentation by TF is compared with the Active Contour Without Edges (ACWE) [9] and Gradient Vector Flow Snake (GVFS) [2]. The evaluation is done on a harmonic object with varying normal distributed noise $N\left(\mu, \sigma^{2}\right)$, as shown at the top row in Fig. 5. The sum of squared error (SSE) is employed to quantify the performance of each algorithm.

$$
S S E=\sum_{i=1}^{M} \sum_{j=1}^{N}\left(S_{i, j}-A_{i, j}\right)^{2}
$$

Where, $S$ is the binary segmented image and $A$ is the actual binary segmented image of size $M \times N$. The quantity of noise is considered in terms of standard deviation $\sigma$ with zero mean.

ACWE is a region based GAC model that is implemented with a level set function. It applies global minimization to especially segment bimodal images as a whole. However, in this evaluation, we choose the biggest segmented region, since we are concerned with the harmonic object segmentation. Otherwise, it will cause very high errors in noisy conditions because of the segmented noises outside the harmonic object. In this evaluation, the selected parameter values for ACWE are: $\lambda_{1}=\lambda_{2}=1$ (parameters for regional statistics), $v=0$ (the area parameter), $h=1$ (the step space), $\Delta t=0.1$ (the time space), $\varepsilon=1$ (the parameter for the Heaviside and Dirac delta functions) and $\mu=0.1 * 255^{2}$ (the length parameter).

GVFS is a gradient based PAC model that uses GVF as an external force. In this evaluation, the selected parameter values for GVFS are: $\alpha=0.25$ (smoothness of the 
contour), $\beta=0$ (rigidity of the contour) and $\mu=0.2$ (in calculating GVF), $\Delta t=1$ (the time interval). In addition, we use 80 iterations to diffuse gradient vectors.

In our algorithm, we use an explicit scheme of finite differences in the first and in the second part. In this evaluation, the selected parameter values for TF are: $\alpha=0.25$ (thermal diffusivity), $\lambda_{1}=\lambda_{2}=1$ (parameters for regional statistics), $Q=5$ (the energy generated from the source position per unit time interval), $\Delta t=1$ (the time interval), $\Delta x=\Delta y=1$ (the spatial intervals), $t_{s}=10$ (specified time for GHF). In addition, we start to use regional statistics after $t=10$ to increase the number of samples inside of $\mathrm{TF}$ and in each 10 iterations we control the movement of TF to determine the termination of the first part.

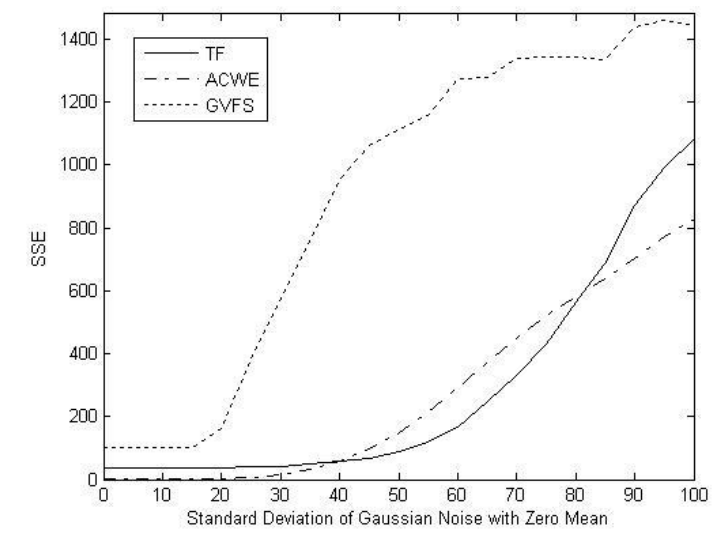

Fig. 4. Performance of TF, ACWE and GVFS

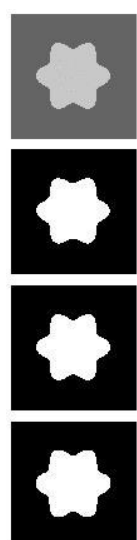

(a) $\sigma=0$
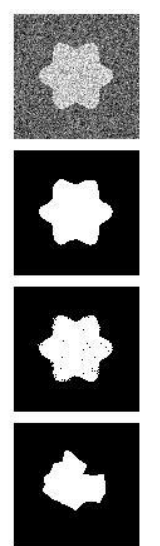

(b) $\sigma=40$
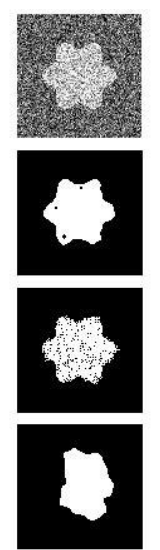

(c) $\sigma=60$
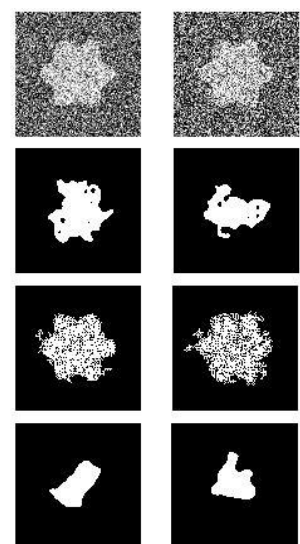

$\begin{array}{ll}\text { (d) } \sigma=80 & \text { (e) } \sigma=100\end{array}$
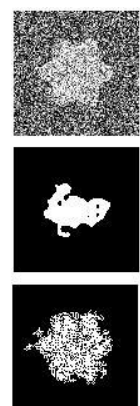

Fig. 5. Results for TF (second row), ACWE (third row) and GVFS (forth row) with respect to increasing Gaussian noise in the image of size $100 \times 100$ 
In this evaluation, the contours and the heat source are initialized inside the harmonic object. Fig. 4 shows performance of TF, ACWE and GVFS. It is observed that TF and ACWE perform much better than GVFS. The reason for this is that TF and ACWE use region based algorithms, on the other hand GVFS uses a gradient based algorithm, which is very sensitive to the noisy conditions. When we compare TF and ACWE, ACWE performs better than TF until $\sigma \cong 40$. This appears to be due to the smoothing operation in TF. GHF attempts to smooth the original shape and cause errors in TF, when there is no noise or low noise in the image, since $t_{s}$ is fixed in the evaluation. However, from $\sigma \cong 40$ to $\sigma \cong 80$, TF segments better than ACWE. The main reason is again the smoothing operation. TF applies smoothing after rough segmentation without any relation to the regional statistic constraints, while ACWE uses smoothness constraint with regional statistic constraints during the segmentation. After $\sigma \cong 80$, it is seen that ACWE shows better performance than TF. Because, ACWE segments many regions outside the harmonic region in the presence of high noise and then some of the segmented noise remains connected to the original region when we select the biggest region. Fig. 5 shows some of the results for TF (second row), ACWE (third row) and GVFS (forth row).

Simulation results also show the effectiveness and the computational efficiency of our algorithm in comparison to the GVFS and ACWE. All the evaluations and the simulation results are obtained by using MATLAB 7.0 on a Pentium IV computer, which runs Windows XP operating system with $3.2 \mathrm{GHz}$ CPU and 1GB RAM. Fig. 6

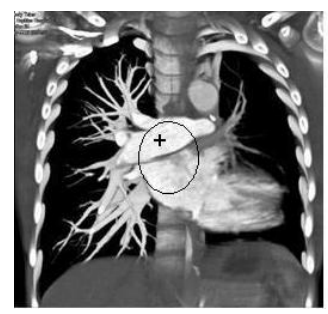

(a)

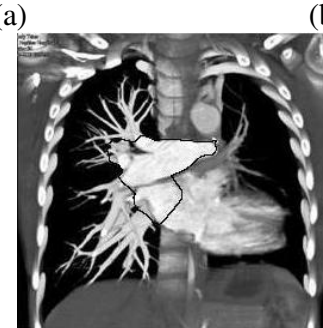

(d)

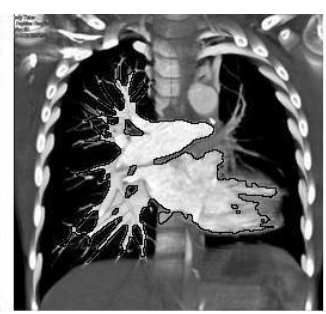

(b)

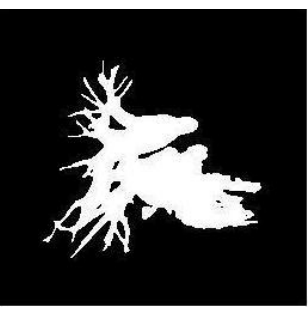

(c)

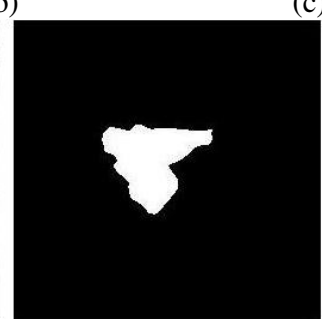

(e)

Fig. 6. Segmentation of pulmonary arterial branches in the chest image of size $259 \times 250$ by TF and GVFS. (a) Initial contour and the source position. (b) Segmentation by TF is shown by the black contour on the image. All the parameters are same as in evaluation except $t_{s}=5$. (CPU=7.85 second). (c) Segmentation by TF is in binary form. (d) Segmentation by GVFS is shown with black contour on the image. All the parameters are same as in evaluation except the iteration to diffuse gradient vectors is 70 . (CPU=9.23 second). (e) Segmentation by GVFS is in binary form. 


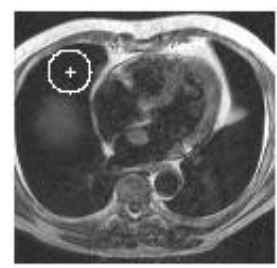

(a)

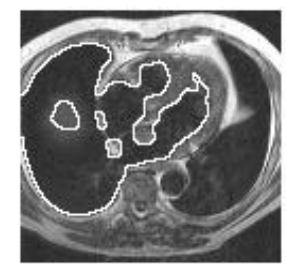

(b)

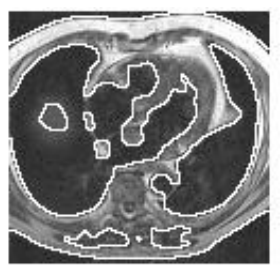

(c)

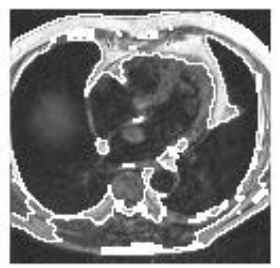

(d)

Fig. 7. Segmentation of human lung image of size $123 \times 118$ by TF, CF and ACWE. (a) Initial contour and the source position. (b) Segmentation by TF is shown with white contour on the image. All the parameters are same as in evaluation except $t_{s}=15$ (CPU=1.96 second). (c) Segmentation by CF. $t_{s}=15$. (CPU=1.96 second). (d) Segmentation by ACWE. All the parameters are same as in evaluation except the length parameter $\mu=0.08 \times 255^{2}$. (CPU $=15.92$ minutes).

shows the segmentation of pulmonary arterial branches in the chest image by TF and GVFS. The initial contour for GVFS and the source position for TF are shown in Fig. 6(a). Fig. 6(b) shows the segmentation by TF in the given image with black contour, however the segmented arterial branches are not visible with this illustration and the segmentation is also shown with the binary form in Fig. 6(c). On the other hand, Fig. 6(d) and (e) show segmentation by GVFS respectively with black contour in the image and the binary form. It is observed that TF segments the desired arterial branches better than GVFS. By this result, TF can easily handle topological changes and flow into the arterial branches with CPU=7.85s. However, GVFS cannot handle topological changes and cannot flow into the arterial branches. Although GVFS segments a smaller region than $\mathrm{TF}$, the $\mathrm{CPU}=9.23 \mathrm{~s}$, which is more than for $\mathrm{TF}$.

Fig. 7 shows the segmentation of bimodal human lung image by TF, CF and ACWE, where the initial contour for ACWE and the source position for TF are shown in Fig. 7(a). Fig. 7(b) and (c) respectively show the segmentation by TF and CF with white contour in the image. Fig. 7(d) shows the segmentation by ACWE. It is observed that $\mathrm{TF}$ and $\mathrm{CF}$ achieves segmentation with $\mathrm{CPU}=1.96$ seconds and ACWE achieves with CPU=15.92 minutes. This big difference in CPU time appears because of the computational complexity of ACWE that is implemented with level sets. It is also observed that CF can extract feature boundaries better than ACWE especially at the middle and at the bottom of the lung image.

\section{Conclusions}

We have presented a novel segmentation algorithm based on heat flow analogy. In the first part of the algorithm, we roughly extract the desired feature boundaries by representing particular heat conduction problem in the image domain. The representation in image domain is achieved by using a control function $(\mathrm{CF})$ in the heat conduction equation. This formulation also provides advantage when the given image is bimodal, since $\mathrm{CF}$ attempts to segment whole image in this case. In the second part, we use geometric heat flow (GHF) to tune the curvature of the extracted feature boundaries 
and remove possible noise that arises from the first part of the segmentation. Evaluation results indicate that temperature front (TF) has better performance than gradient vector flow snake (GVFS) and active contour without edges (ACWE) with respect to increasing Gaussian noise. For the bimodal images, TF and CF are again more efficient and effective than both GVFS and ACWE based on the simulation results. As such, the heat analogy can be deployed with success for shape extraction in images.

\section{References}

1. Kass, M., Witkin, A., Terzopoulos, D.: Snakes: Active Contour models. In: IJCV, pp. 321-331 (1987)

2. Xu, C., Prince, J.L.: Snakes, Shapes and Gradient Vector Flow. IEEE Transaction on Image Processing 7(3), 359-369 (1998)

3. Caselles, V., Catte, F., Coll, T., Dibos, F.: A Geometric Model for Active Contours. Numerische Mathematic 66, 1-31 (1993)

4. Malladi, R., Sethian, J.A., Vemuri, B.C.: Shape Modeling with Front Propagation: A Level Set Approach. IEEE Transaction on PAMI 17(2), 158-175 (1995)

5. Caselles, V., Kimmel, R., Sapiro, G.: Geodesic Active Contours. IJCV 22(1), 61-79 (1997)

6. Adalsteinsson, D., Sethian, J.: A Fast Level Set Method for Propagating Interfaces. J. Computational Physics 118(2), 269-277 (1995)

7. Sethian, J.: Level Set Methods and Fast Marching Methods. Cambridge Univ. press, New York (1999)

8. Weickert, J., Bart, M., Romeny, T.H., Viergever, M.A.: Efficient and Reliable Schemes for Nonlinear Diffusion Filtering. IEEE Transaction on Image Processing 7(3), 398-410 (1998)

9. Chan, T., Vese, L.: Active Contours without Edges. IEEE Transaction on Image Processing 10(2), 266-277 (2001)

10. Mumford, D., Shah, J.: Optimal Approximation by Piecewise Smooth Functions and Associated Variational Problems. Comm. Pure and Applied Math. 42, 577-685 (1989)

11. Adams, R., Bischof, L.: Seeded region growing. IEEE Trans. PAMI 16(6), 641-647 (1994)

12. Fung, P.W., Grebbin, G., Attikiouzel, Y.: Model-based region growing segmentation of textured images. In: ICASSP-90, vol. 4, pp. 2313-2316 (1990)

13. Perona, P., Malik, J.: Scale-Space and Edge Detection using Anisotropic Diffusion. IEEE Trans. PAMI 22(8), 629-639 (1990)

14. Kimia, B.B., Siddiqi, K.: Geometric Heat Equation and Nonlinear Diffusion of Shapes and Images. In: CVPR, pp. 113-120 (1994)

15. Direkoğlu, C., Nixon, M.S.: Low Level Moving-Feature Extraction via Heat Flow Analogy. In: Bebis, G., Boyle, R., Parvin, B., Koracin, D., Remagnino, P., Nefian, A., Meenakshisundaram, G., Pascucci, V., Zara, J., Molineros, J., Theisel, H., Malzbender, T. (eds.) ISVC 2006. LNCS, vol. 4291, pp. 243-252. Springer, Heidelberg (2006)

16. Acton, S.T., Bovik, A.C., Crawford, M.M.: Anisotropic diffusion pyramids for image segmentation. In: ICIP (1994)

17. Manay, S., Yezzi, A.: Anti-Geometric Diffusion for Adaptive Thresholding and Fast Segmentation. IEEE Transaction on Image Processing 12(11) (2003)

18. Holman, J.P.: Heat Transfer, 9th edn. McGraw-Hill, New York (2002) 